\title{
Frequency-Stabilized Source of Single Photons from a Solid-State Qubit
}

\author{
Jonathan H. Prechtel, ${ }^{1, *}$ Andreas V. Kuhlmann, ${ }^{1}$ Julien Houel, ${ }^{1,2}$ Lukas Greuter, ${ }^{1}$ Arne Ludwig, ${ }^{3}$ Dirk Reuter, ${ }^{3,4}$ \\ Andreas D. Wieck, ${ }^{3}$ and Richard J. Warburton ${ }^{1}$ \\ ${ }^{1}$ Department of Physics, University of Basel, Klingelbergstrasse 82, CH-4056 Basel, Switzerland \\ ${ }^{2}$ Bâtiment Alfred Kastler, Université Claude Bernad, F-69622 Lyon Villeurbanne, France \\ ${ }^{3}$ Lehrstuhl für Angewandte Festkörperphysik, Ruhr-Universität Bochum, Universitätsstrasse 150, D-44780 Bochum, Germany \\ ${ }^{4}$ Department Physik, Universität Paderborn, Warburgerstrasse 100, D-33098 Paderborn, Germany
}

(Received 3 July 2013; published 23 October 2013)

\begin{abstract}
Single quantum dots are solid-state emitters that mimic two-level atoms but with a highly enhanced spontaneous emission rate. A single quantum dot is the basis for a potentially excellent single-photon source. One outstanding problem is that there is considerable noise in the emission frequency, making it very difficult to couple the quantum dot to another quantum system. We solve this problem here with a dynamic feedback technique that locks the quantum-dot emission frequency to a reference. The incoherent scattering (resonance fluorescence) represents the single-photon output, whereas the coherent scattering (Rayleigh scattering) is used for the feedback control. The fluctuations in emission frequency are reduced to $20 \mathrm{MHz}$, just approximately $5 \%$ of the quantum-dot optical linewidth, even over several hours. By eliminating the $1 / f$-like noise, the relative fluctuations in quantum-dot noise power are reduced to approximately $10^{-5}$ at low frequency. Under these conditions, the antibunching dip in the resonance fluorescence is described extremely well by the two-level atom result. The technique represents a way of removing charge noise from a quantum device.
\end{abstract}

DOI: 10.1103/PhysRevX.3.041006

Single photons are ideal carriers of quantum information [1-3]. A quantum state stored in one of the degrees of freedom of the photon's wave packet (polarization, phase, or time bin) can be maintained over long distances. Single photons are therefore important in quantum communication [3], for coupling remote stationary qubits [4], the basis of a quantum repeater [5], or for coupling different elements in a quantum device. Furthermore, single photons are the seed for a variety of quantum optics experiments [6,7].

Key parameters for a single-photon source are fidelity of the antibunching, flux, wavelength, and photon indistinguishability [8]. Remarkably, solid-state emitters are presently better able to meet these demands than atomic systems [6,7]. In particular, spontaneous emission from individual quantum dots embedded in an inorganic semiconductor is a very promising source of highly antibunched, high-flux, indistinguishable photons [7,9,10]. The antibunching, particularly with resonant excitation, is very high [11]. The radiative lifetime is very short, typically just less than $1 \mathrm{~ns}[12]$. The flux is usually limited by the poor collection efficiency: Most of the light is internally reflected at the GaAs-vacuum interface. However, this problem can be solved by nanostructuring the photonic modes to create a microcavity [13] or a

\footnotetext{
*jonathan.prechtel@unibas.ch; http://nano-photonics.unibas.ch/
}

Published by the American Physical Society under the terms of the Creative Commons Attribution 3.0 License. Further distribution of this work must maintain attribution to the author(s) and the published article's title, journal citation, and DOI.
Subject Areas: Nanophysics, Photonics, Quantum Physics

photonic nanowire [14]. In the latter case, collection efficiencies of approximately $70 \%$ have been achieved. The photon indistinguishability is very high for successive photons [10]. Based on the optical linewidth, typically a factor of 2 above the transform limit when measured with resonant excitation [15-18], the indistinguishability is also reasonably high for photons emitted widely separated in time. Furthermore, a single quantum dot has also been developed as a spin qubit [19], facilitating an interface between stationary qubits and photons [20-22].

Unlike a real atom, the exact transition wavelength of a quantum dot is not locked to any particular wavelength and varies considerably from quantum dot to quantum dot. However, the host semiconductor can be designed so that considerable possibilities for tuning the emission wavelength exist. Electric-field tuning [23,24] and strain tuning $[25,26]$ allow the emission wavelength to be tuned over several nanometers. A major problem remains. The emission wavelength is not constant: It varies randomly over time, even in very controlled environments at low temperature. The culprit at low frequency is electrical noise in the semiconductor that shifts the emission wavelength via the Stark effect [18]. This noise has a $1 / f$-like power spectrum, resulting in, first, large and uncontrolled drifts at low frequencies and, second, an undefined mean value. This noise, while poorly understood, is ubiquitous in semiconductors and makes it very difficult to couple an individual quantum dot to another quantum system, another quantum dot, for instance, or an ensemble of cold atoms. We present here a new scheme that solves this problem: We create a stream of single photons with a wavelength that remains constant even over several hours. 
The output of our quantum device is a stream of single photons generated by resonance fluorescence (RF) from a single quantum dot. RF has considerable advantages over nonresonant excitation of photoluminescence: The linewidth is much lower [17,18], and the antibunching is much better. We lock the wavelength of the quantum device to a stable reference. We generate an error signal, a signal with large slope at its zero crossing, by measuring the differential transmission $\Delta T / T$ simultaneously $[15,27,28]$. The control variable is the voltage $V_{g}$ applied to a surface gate that influences the quantum-dot frequency via the Stark effect. The performance of the feedback scheme is characterized by, first, measuring a series of snapshots of the optical resonance to assess the residual frequency jitter and, second, by carrying out a full analysis of the noise in the RF.

Our scheme goes well beyond previous attempts at single-emitter stabilization in the solid state $[29,30]$. The first experiment on frequency stabilization locked a nonstandard quantum dot at $780 \mathrm{~nm}$ to the atomic resonance of $\mathrm{Rb}$ [29]. We are not limited to any "magic" wavelengths, and, in particular, we can stabilize the emission wavelengths of high-quality InGaAs quantum dots that typically emit in the 900-1000-nm range. The second advance of our scheme is a 100 times better frequency stabilization relative to Ref. [29]. Here, the absolute frequency of the quantum-dot emission is locked with an uncertainty of just $20 \mathrm{MHz}$. We observe a reduction in the noise power up to a frequency of approximately $100 \mathrm{~Hz}$, a bandwidth high enough to eliminate the substantial drifts at low frequency.

A sketch of the experimental concept is shown in Fig. 1(a). A linearly polarized resonant laser is focused onto the sample surface and drives the optical transition. The resonance fluorescence of the quantum dot is collected with a polarization-based dark-field technique [17,20,31], described in detail elsewhere [32]. Simultaneously, the optical resonance is detected in transmission by superimposing a sublinewidth modulation to the gate. The transmission signal arises from an interference of quantum-dot scattering with the driving laser [28]. The incoherent part, i.e., the resonance fluorescence, averages to zero in transmission; what is detected instead is the coherent scattering, i.e., the Rayleigh scattering. In this way, the experiment utilizes both incoherent and coherent parts of the scattered light, for the singlephoton output and control, respectively. With a small modulation, the transmission signal has a large slope with zero crossing at zero detuning and is therefore ideal for the
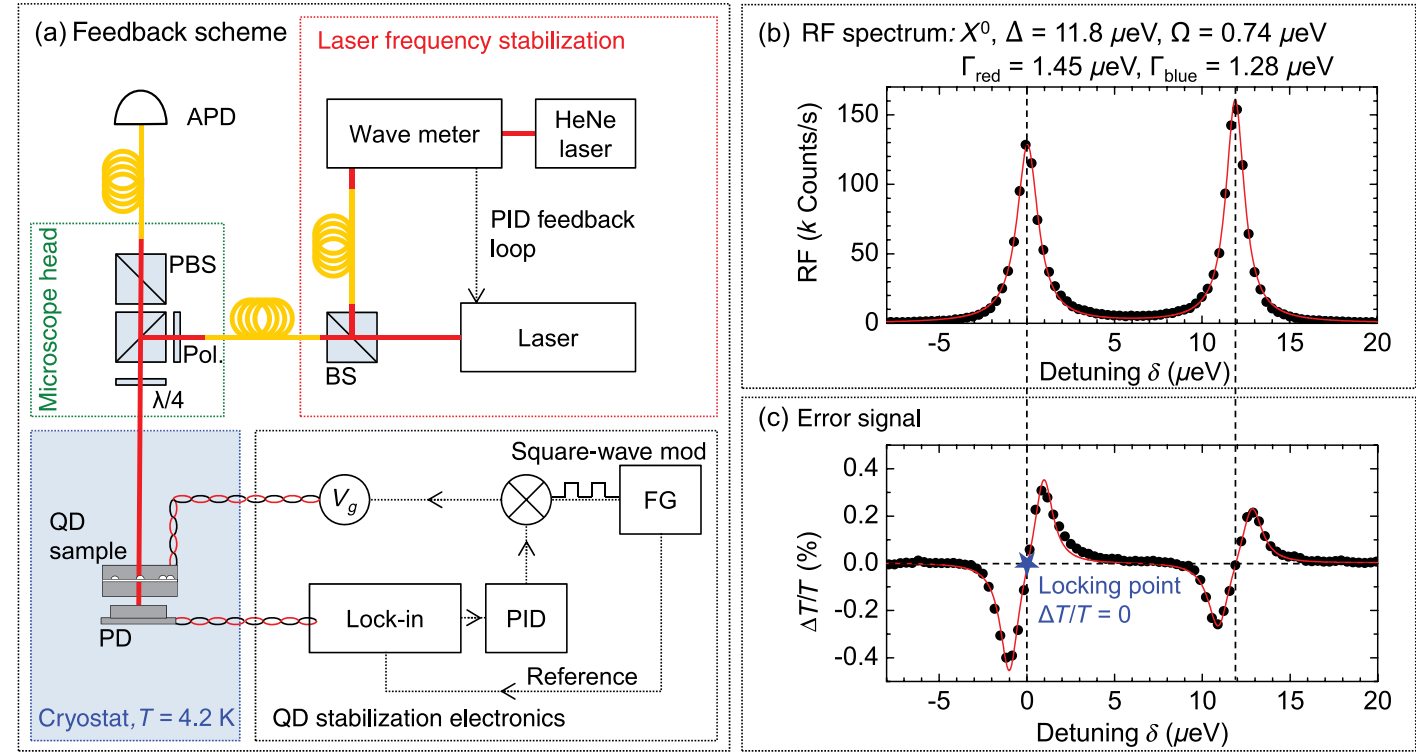

FIG. 1. (a) Schematic view of the experiment. The narrow-band laser is stabilized to a fixed frequency by a wave meter which in turn is stabilized to a HeNe laser. Laser light is guided through optical fibers (yellow curves) and microscope optics before it is focused onto the sample, driving the $X^{0}$ transition resonantly (BS = beam splitter, PBS = polarizing BS, and Pol. = linear polarizer). Two simultaneous measurements of $X^{0}$ scattering are performed: RF, detected with an APD, and absorption with a photodiode (PD) underneath the sample. The dynamic stabilization is realized with an active PID feedback loop that corrects for fluctuations in the transition energy using the gate voltage $V_{g}$ and the square-wave modulation of a function generator (FG). (b) RF signal of the finestructure split $X^{0}$ emission of a single quantum dot at wavelength $936.5 \mathrm{~nm}$, a power corresponding to a Rabi energy $\Omega$ of $0.74 \mu \mathrm{eV}$ and a temperature of $4.2 \mathrm{~K}$. A detuning is achieved by sweeping the gate voltage. The solid red line is a Lorentzian fit to the data with linewidth $\Gamma=1.28 \mu \mathrm{eV}(309 \mathrm{MHz})$ and $\Gamma=1.45 \mu \mathrm{eV}$ (350 MHz) and with a fine-structure splitting $\Delta=11.8 \mu \mathrm{eV}$. (c) The differential transmission $(\Delta T / T)$ signal on the same quantum dot with integration time $100 \mathrm{~ms}$ per point. The red curve is a fit to the derivative of the two Lorentzians. The signal around the zero-crossing point $(\Delta T / T=0)$ is used to generate an error signal for the feedback scheme. 
generation of an error signal. $\Delta T / T$, the error signal, is recorded with a lock-in amplifier to reject noise, and the lock-in output is fed into a classical feedback scheme. The feedback output is, like the modulation, applied to the gate electrode of the device. The set point of the control loop is the zero crossing with the goal of locking the peak of the quantum-dot RF spectrum to the laser. The laser itself is locked to a HeNe laser reference.

The self-assembled InGaAs quantum dots, grown by molecular beam epitaxy, are integrated into a semiconductor charge-tunable heterostructure [33]. The quantum dots are located $25 \mathrm{~nm}$ above a heavily $n$-doped GaAs back contact $\left(n=1.7 \times 10^{18} \mathrm{~cm}^{-3}\right)$. The intermediate layer, undoped GaAs $(25 \mathrm{~nm})$, acts as a tunneling barrier. A 150-nm GaAs layer caps the quantum dots, and an AlAs/ GaAs superlattice ( 68 periods of AlAs/GaAs $3 \mathrm{~nm} / 1 \mathrm{~nm}$ ) completes the heterostructure. A Ti/Au $(5 \mathrm{~nm} / 10 \mathrm{~nm})$ Schottky gate is deposited on the sample surface; Ohmic contacts are prepared to the back contact. Bias $V_{g}$ is applied between the Schottky gate and the back contact. The sample is placed in a liquid-helium-bath cryostat at $4.2 \mathrm{~K}$ with a residual magnetic field of $10 \mathrm{mT}$.

The single-quantum-dot spectroscopy is performed with a confocal microscope. The continuous-wave laser has a short-term linewidth of $1 \mathrm{MHz}$. Long-term wavelength stability of approximately $2 \mathrm{MHz}$ is achieved by locking the laser to a high-resolution wave meter, itself locked to a low-linewidth (25-MHz) HeNe laser. The size of the focal spot and the collection efficiency of the single-quantumdot RF are both enhanced with a half-sphere zirconia solid immersion lens positioned on top of the Schottky gate. Figure 1(b) shows a RF signal from the neutral exciton transition $|0\rangle \leftrightarrow\left|X^{0}\right\rangle$, where $\left|X^{0}\right\rangle$ represents an electronhole complex and $|0\rangle$ the crystal ground state. The RF is detected with a silicon avalanche photodiode (APD) in single-photon-counting mode, and the detuning of the quantum-dot resonances relative to the constant-frequency laser is achieved in this case with the Stark shift induced by the bias $V_{g}$. The $X^{0}$ exhibits a fine-structure splitting of $11.8 \mu \mathrm{eV}$, the two lines having linewidths $\Gamma=1.45$ and $1.28 \mu \mathrm{eV}$ close to the transform limit of $\Gamma_{0}=\hbar / \tau_{r}=$ $0.93 \mu \mathrm{eV}(220 \mathrm{MHz})$, where $\tau_{r}$ is the radiative lifetime of the exciton transition $\left[\tau_{r}=(0.71 \pm 0.01) \mathrm{ns}\right.$ here $]$.

A sublinewidth square-wave modulation at $527 \mathrm{~Hz}$ is applied to the Schottky gate. The modulation broadens both $X^{0}$ transitions slightly, here the "red" transition from $\Gamma=1.45$ to $\Gamma=2.58 \mu \mathrm{eV}$. The transmitted light is detected with an in situ photodiode connected to a roomtemperature current-voltage preamplifier. Lock-in detection of the $\Delta T / T$ signal is shown in Fig. 1(c). With the sublinewidth modulation, the $\Delta T / T$ resonance is proportional to the derivative of the RF spectrum [27]. There are two points that cross with high slope through zero, one for each $X^{0}$ transition. Both crossing points enable a feedback scheme: $\Delta T / T$ provides the error signal and $V_{g}$ the control parameter. For instance, if the transition energy increases because of electric fluctuations in the sample, $\Delta T / T$ moves away from zero. Once such a change is detected, a modified $V_{g}$ is applied to the gate to bring the resonance back to the set point. For the feedback circuit, we use a proportional, integral, and derivative (PID) control loop. The proportional factor $P=0.1$ is chosen with respect to the slope of the error signal, while the integral $I=0.06$ and the derivative constant $D=6 \times 10^{-5}$ are obtained by tuning methods. The signal-to-noise ratio in the $\Delta T / T$ circuit allows us to run the feedback scheme with a bandwidth up to approximately $50 \mathrm{~Hz}$. The "red" $X^{0}$ transition is used for the subsequent feedback experiments because it has a higher $\Delta T / T$ contrast than the "blue" $X^{0}$ transition.

The noise in the device consists of charge noise and spin noise [18]. The charge-noise power spectrum consists of $1 / f$-like noise and Lorentzian noise, the latter with a characteristic frequency of approximately $10 \mathrm{~Hz}$ [18]. The frequency bandwidth of the feedback is therefore sufficient to eliminate the low-frequency drift and most of the Lorentzian noise. The spin-noise power spectrum has a smaller amplitude but higher characteristic frequency, approximately $10 \mathrm{kHz}$ [18], exceeding the bandwidth of the feedback.

The performance of the single-quantum-dot frequency stabilization is put to the test in a stroboscopic experiment. The $X^{0}$ transition energy is mapped with a second laser (linewidth also $1 \mathrm{MHz}$ ). The first laser stabilizes the transition with the feedback scheme at a power corresponding to a Rabi energy $\Omega$ of $0.74 \mu \mathrm{eV}$. A second laser of identical power is tuned with triangular function back and forth through the same transition with a rate of $8.0 \mu \mathrm{eV} / \mathrm{s}$. The sum of the power of both lasers is selected to lie below the power at which power broadening becomes significant. The RF spectrum is fitted to a constant (to describe RF from the first laser) plus Lorentzian function (to describe RF from the second laser) in order to determine the center position of the resonance. In this way, a "snapshot" of the resonance position is recorded every $5 \mathrm{~s}$ with an "exposure time" of $100 \mathrm{~ms}$ for a total of $1000 \mathrm{~s}$. The distribution of the peak position can be seen in the histogram in Fig. 2. In Figs. 2(a) and 2(b), the scanning laser results in an asymmetry: The resonance frequency is more likely to lie at positive detunings on sweeping from negative to positive detunings, and vice versa. The asymmetry is probably related to the so-called "dragging" effect [34] that is very pronounced on this quantum dot at high magnetic fields (above $0.1 \mathrm{~T}$ ) [32]: The nuclear spins polarize in such a way as to maintain the resonance with the laser over large detunings. In other words, it is likely that the asymmetries in Figs. 2(a) and 2(b) are the first hints of dragging. The histogram in Fig. 2(c) is a combination of the data sets of Figs. 2(a) and 2(b) that are influenced least by dragging (up-sweeps at negative detuning, down-sweeps at positive detuning). Without the 

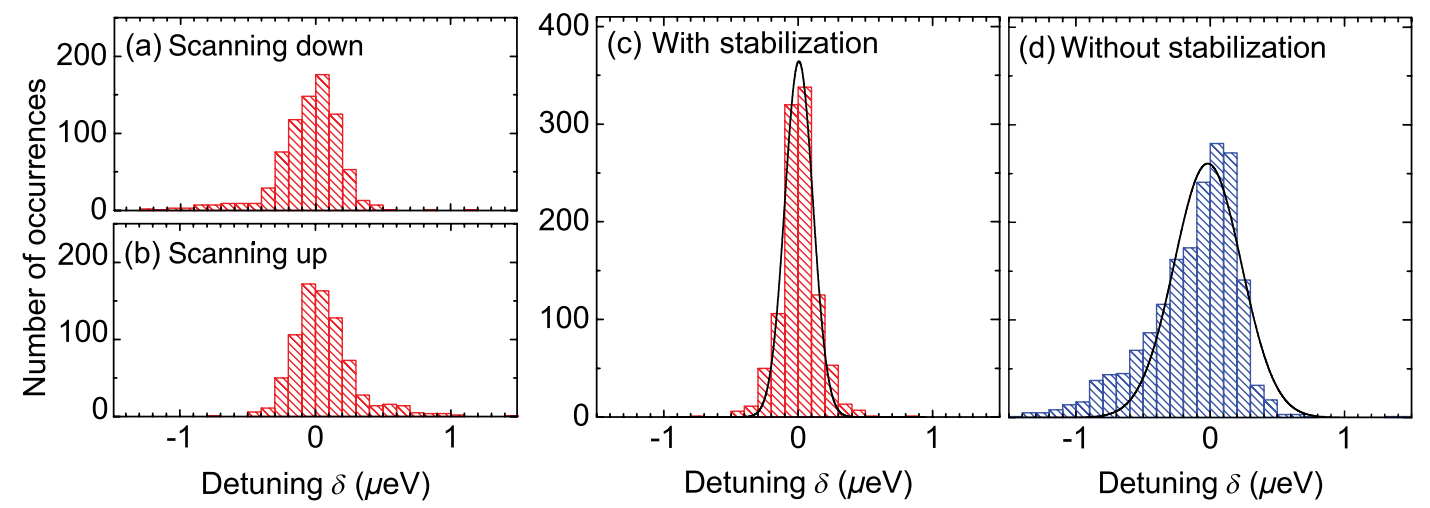

FIG. 2. Histogram of the RF peak position (a)-(c) with and (d) without the stabilization scheme. A triangle $V_{g}$ is applied. The scanning rate of the laser is $8.0 \mu \mathrm{eV} / \mathrm{s}$ with period $10 \mathrm{~s}$. Histograms of the RF peak position for (a) up-sweeps and (b) down-sweeps recorded with feedback. (c) The histogram is shown with feedback, negative detunings from the up-sweeps and positive detunings from the down-sweeps. A histogram without feedback is shown in (d). The standard deviation $\sigma$ is reduced from (d) $0.250 \mu \mathrm{eV}$ $(61 \mathrm{MHz})$ without active stabilization to (c) $0.089 \mu \mathrm{eV}(22 \mathrm{MHz})$ with active stabilization.

stabilizing loop, the long-term drift, i.e., the $1 / f$-like noise, results in a broader distribution; see Fig. 2(d). This drift also leads to the asymmetry in Fig. 2(d), reflecting a trend to the red in this particular case. The fluctuations in resonance positions are quantified with the standard deviation $\sigma_{E}$ of the peak positions. Without stabilization [Fig. 2(d)], $\sigma_{E}^{\mathrm{OFF}}=0.250 \mu \mathrm{eV}(61 \mathrm{MHz})$. With active stabilization, $\sigma=0.102 \mu \mathrm{eV}(25 \mathrm{MHz})$. This value is small enough to be influenced by shot noise in each data point, which results in an energy uncertainty on fitting each spectrum to a Lorentzian. The shot noise results in an energy jitter of $\sigma_{E \text {,shot }}=0.049 \mu \mathrm{eV}$, giving $\sigma_{E}^{\mathrm{ON}}=\sqrt{\sigma^{2}-\sigma_{E \text {, shot }}^{2}}=$ $0.089 \mu \mathrm{eV}(22 \mathrm{MHz}), 36 \%$ of $\sigma_{E}^{\text {OFF }}$. The measurement of $\sigma_{E}$ represents a measurement of the noise in a bandwidth from approximately $1 \mathrm{mHz}$ to approximately $3.1 \mathrm{~Hz}$. (Noise at higher frequencies is reflected in the linewidth $Г$.) The ratio $\sigma_{E}^{\mathrm{OFF}}: \sigma_{E}^{\mathrm{ON}}$ would increase if lower frequencies were included on account of the $1 / f$-like noise: $\sigma_{E}^{\mathrm{ON}}$ would remain the same, but $\sigma_{E}^{\mathrm{OFF}}$ would increase.

The ultimate operation capability of the stabilization system is limited by the random noise in the output of the PID electronics. In Fig. 1(c), the noise in the $\Delta T / T$ signal is $\sigma_{\Delta T / T}=1.45 \times 10^{-4}$. In the ideal case, the transmission noise determines the energy jitter of the quantum-dot resonance position [35]

$$
\sigma_{E, \min }=\frac{d \delta}{d \Delta T} \sigma_{\Delta T / T} \simeq 0.013 \mu \mathrm{eV} \quad(3 \mathrm{MHz}),
$$

where $\delta$ is the detuning. This limit, approximately 100 times smaller than the linewidth, illustrates the power of this technique. We have not yet reached this limit in practice. Nevertheless, stabilization with a residual jitter down to just $\sigma_{E} / h=22 \mathrm{MHz}$ is achieved.

The frequency-locking feedback scheme is also tested regarding its long-term behavior and bandwidth. The RF signal is recorded over several hours [Fig. 3(a)] without (blue) and with (red) the stabilizing loop. The measurements
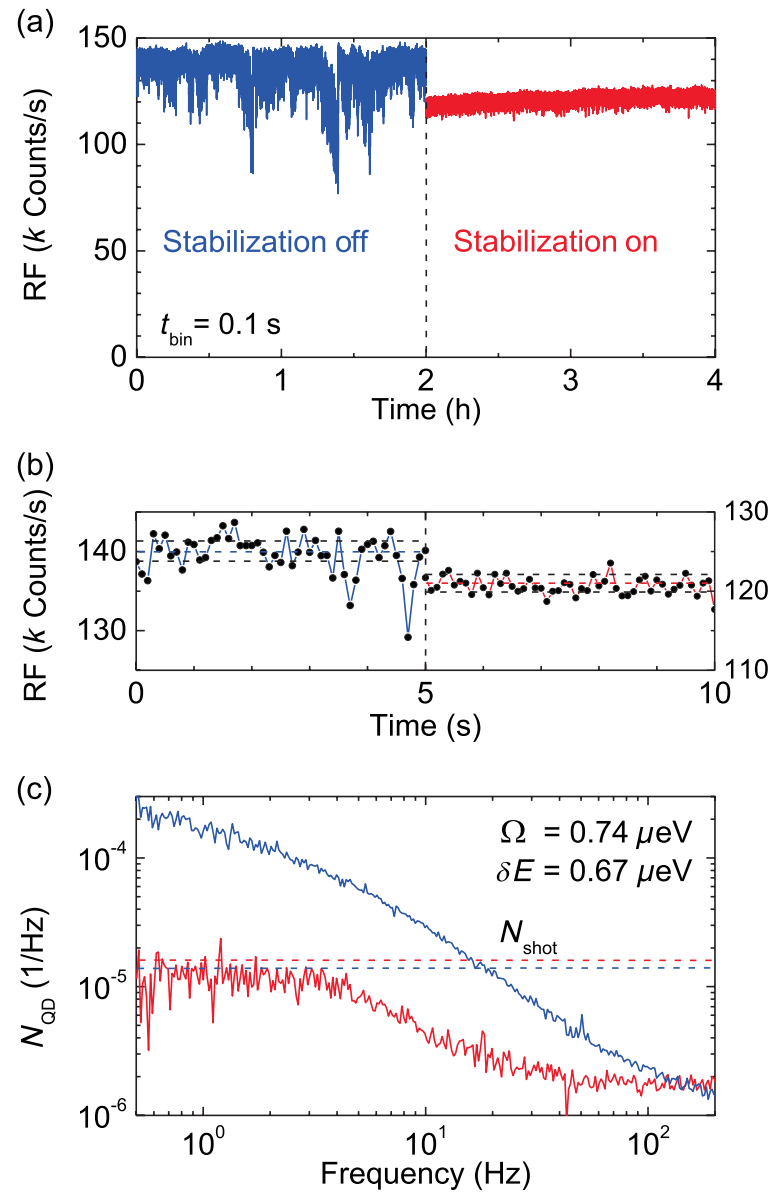

FIG. 3. (a) Time trace of the RF of a single quantum dot (the one from Fig. 1) with $\delta=0 \mu \mathrm{eV}$ recorded over several hours. The binning time is $t_{\text {bin }}=100 \mathrm{~ms}$. The time trace is plotted with (red) and without (blue) the dynamic stabilization scheme. (b) 5-s excerpts of the unstabilized (blue) and stabilized (red) time traces, with the dashed lines representing the shot-noise limits. (c) Noise spectra of the normalized RF signal $S(t) /\langle S(t)\rangle$ corresponding to the time traces of (a) after correction for external noise sources. The shot noise in the experiment is shown with the dashed lines. 
are accomplished by tuning the $X^{0}$ of the quantum dot via the Stark effect into resonance with the excitation laser $(\delta=0 \mu \mathrm{eV})$ and then recording the arrival time of each single photon detected by the APD over the duration of the entire experiment $T$. Postexperiment, the data are analyzed by setting a binning time, $t_{\text {bin }}=100 \mathrm{~ms}$ in this case. For a fixed $V_{g}$, the RF counts show large fluctuations up to a factor of 2 (blue curve). The origins are slow electrical fluctuations in the sample that cause the transition to drift out of resonance with the laser. With the feedback on, these fluctuations disappear and the RF remains at a constant level (red curve). The fluctuations in the red curve arise almost entirely from shot noise in the detector [Fig. 3(b)]. The average RF signal is a little smaller with feedback because the applied modulation slightly broadens the resonance.

Insight into the bandwidth of the stabilization mechanism is revealed by a fast Fourier transform (FFT) of the time trace. Although the shot noise dominates, the shot noise can be independently measured with a small amount of reflected laser light as a source, allowing us to determine the noise coming solely from the quantum dot. The FFT of the normalized RF signal $S(t) /\langle S(t)\rangle$ provides a noise spectrum [18]:

$$
N_{\mathrm{RF}}(f)=|\operatorname{FFT}[S(t) /\langle S(t)\rangle]|^{2}\left(t_{\text {bin }}\right)^{2} / T .
$$

For $N_{\mathrm{RF}}(f), t_{\mathrm{bin}}=1 \mu \mathrm{s}$ and $T=2 \mathrm{~h}$. The noise spectrum of the quantum dot $N_{\mathrm{QD}}(f)$ is obtained by correcting the RF noise by the noise of the experiment $N_{\text {exp }}(f)\left[N_{\mathrm{QD}}(f)=\right.$ $\left.N_{\mathrm{RF}}(f)-N_{\text {exp }}(f)\right] . N_{\mathrm{QD}}(f)$ corresponding to the time traces of Fig. 3(a) are shown in Fig. 3(c). Without feedback, $N_{\mathrm{QD}}(f)$ has a $1 / f$-like dependence on $f$ as a consequence of charge noise in the device. With feedback, $N_{\mathrm{QD}}(f)$ is reduced by up to a factor of 20 at the lowest frequencies and is constant: The $1 / f$-like noise is eliminated. The two curves meet at $f \simeq 130 \mathrm{~Hz}$ once the bandwidth of the PID circuit has been exceeded. At higher frequency, the noise spectrum is dominated by spin noise [18].

The two experiments, intensity-noise and energy-jitter measurements, can be linked to add weight to our analysis. Specifically, we forge a relationship between the RF noise under feedback and the jitter in the energy detuning $\sigma_{E}$, connecting a measurement of noise in a time trace to a separate measurement of a fluctuation in an energy detuning. The detuning jitter is much less than the linewidth, such that the change in the RF signal $(\Delta \mathrm{RF})$ is related quadratically to the detuning for fluctuations around $\delta=0$. The variance of the RF noise $\sigma_{\mathrm{RF}}^{2}$ is related to an integral of the noise curve $\sigma_{\mathrm{RF}}^{2}=\int N_{\mathrm{QD}}(f) d f$ [36]. Integrating up to frequency $\Delta f$ in the regime where $N_{\mathrm{QD}}(f)$ is approximately constant,

$$
\sigma_{E}^{\mathrm{ON}}=\frac{\Gamma}{2}\left(\frac{N_{\mathrm{QD}}(0) \Delta f}{3}\right)^{1 / 4} .
$$

With $\Delta f=3.1 \mathrm{~Hz}, N_{\mathrm{QD}}(0)=1.0 \times 10^{-5}$, and $\Gamma=2.58 \mu \mathrm{eV}$, the equation predicts $\sigma_{E}^{\mathrm{ON}}=0.073 \mu \mathrm{eV}$ that is in excellent agreement with the measurement from the stroboscopic experiment $(0.089 \mu \mathrm{eV})$.

An intensity correlation measurement $g^{(2)}(t)$ is performed with a Hanbury Brown-Twiss interferometer. Low noise $g^{(2)}(t)$ can only be determined at these count rates $(50 \mathrm{kHz}$ per APD) by integrating over several hours, and the feedback is therefore important to ensure that the detuning of the quantum dot with respect to the laser remains constant. $g^{(2)}(t)$ is shown in Fig. 4 from $X^{0}$ of the same quantum dot with zero detuning. $g^{(2)}(t)$ falls to $10 \%$ at $t=0$. This result does not reflect $g^{(2)}(0)$ of the quantum dot but rather the timing jitter of the detectors, which is comparable to the radiative lifetime. We attempt to describe $g^{(2)}(t)$ with a convolution of $g^{(2)}(t)$ for an ideal two-level atom $g_{\text {atom }}^{(2)}(t)$ and the response of the detectors $G(t)$ :

$$
g^{(2)}(t)=g_{\text {atom }}^{(2)}(t) \otimes G(t) .
$$

The detector response is a Gaussian function

$$
G(t)=\frac{1}{\sqrt{2 \pi} \sigma_{D}} \exp \left(-\frac{t^{2}}{2 \sigma_{D}^{2}}\right)
$$

$g_{\text {atom }}^{(2)}(t)$ of a two-level system with resonant excitation is [37]

$g_{\text {atom }}^{(2)}(t)=1-\left[\cos (\lambda t)+\frac{3}{4 \tau_{r}} \lambda \sin (\lambda t)\right] \exp \left(-\frac{3 t}{4 \tau_{r}}\right)$,

with $\lambda=\left[\Omega^{2}-\left(1 / 4 \tau_{r}\right)^{2}\right]^{1 / 2}$ [37]. The temporal jitter of the detector $\tau_{D}=0.40 \mathrm{~ns}$ is measured independently. $\Omega$ and $\tau_{r}$ are known from other experiments to within $10 \%-20 \%$ and are allowed to vary in these windows by a fit routine. The convolution provides an excellent description of the measured $g^{(2)}(t)$ with $\Omega=(0.99 \pm 0.1) \mu \mathrm{eV}$ and $\tau_{r}=(0.78 \pm 0.05) \mathrm{ns}$. In particular, with low systematic error, we can set an upper bound to the quantum dot $g^{(2)}(0)$ of $1 \%-2 \%$.

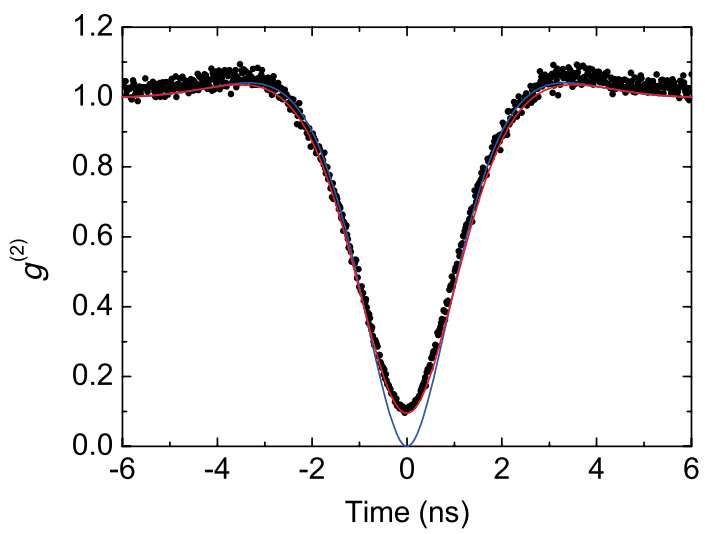

FIG. 4. Second-order correlation $g^{2}(t)$ for the stabilized RF from the $X^{0}$ (black points). The red curve shows a convolution of the two-level atom result with a Gaussian distribution that describes the timing jitter of the detectors. The blue curve shows the two-level atom response alone. 
In conclusion, we have developed a dynamic method of locking the optical resonance of a single quantum dot to a stabilized laser in order to produce a stream of frequencystabilized single photons via resonance fluorescence. Generally speaking, the scheme represents a way to reduce the local charge noise in a semiconductor.

Now that the basic principle is established, there are options for improving the feedback scheme. First, the remaining jitter in the quantum-dot resonance position can be reduced by reducing the noise in the transmission detection. Presently, we are far from the limit defined by the shot noise in the detector current. With lower noise, the feedback bandwidth can also be increased. The tantalizing prospect is to create transform-limited linewidths routinely with high-bandwidth feedback. A bandwidth of about $50 \mathrm{kHz}$ is required [18]. Second, the modulation required here to generate the error signal could be eliminated in a number of ways. For instance, a dispersive line shape can arise naturally in reflectivity via weak coupling to a cavity [38], or the Faraday effect in a small magnetic field [39] could be used.

We acknowledge support from the Swiss National Science Foundation (SNF) and NCCR QSIT. A. L., D. R., and A.D.W. gratefully acknowledge support from DFG SPP1285 and BMBF QuaHLRep 01BQ1035.

[1] Charles H. Bennett and Gilles Brassard, in Proceedings of the IEEE International Conference on Computers, Systems, and Signal Processing, Bangalore, India, 1984 (IEEE, New York, 1984), p. 175-179.

[2] Artur K. Ekert, Quantum Cryptography Based on Bell's Theorem, Phys. Rev. Lett. 67, 661 (1991).

[3] Nicolas Gisin, Grégoire Ribordy, Wolfgang Tittel, and Hugo Zbinden, Quantum Cryptography, Rev. Mod. Phys. 74, 145 (2002).

[4] H. Bernien, B. Hensen, W. Pfaff, G. Koolstra, M. S. Blok, L. Robledo, T. H. Taminiau, M. Markham, D. J. Twitchen, L. Childress, and R. Hanson, Heralded Entanglement between Solid-State Qubits Separated by Three Metres, Nature (London) 497, 86 (2013).

[5] Nicolas Sangouard, Christoph Simon, Hugues de Riedmatten, and Nicolas Gisin, Quantum Repeaters Based on Atomic Ensembles and Linear Optics, Rev. Mod. Phys. 83, 33 (2011).

[6] Sonia Buckley, Kelley Rivoire, and Jelena Vučković, Engineered Quantum Dot Single-Photon Sources, Rep. Prog. Phys. 75, 126503 (2012).

[7] Andrew J. Shields, Semiconductor Quantum Light Sources, Nat. Photonics 1, 215 (2007).

[8] Brahim Lounis and Michel Orrit, Single-Photon Sources, Rep. Prog. Phys. 68, 1129 (2005).

[9] P. Michler, A. Kiraz, C. Becher, W. V. Schoenfeld, P. M. Petroff, Lidong Zhang, E. Hu, and A. Imamoglu, A Quantum Dot Single-Photon Turnstile Device, Science 290, 2282 (2000).
[10] C. Santori, D. Fattal, J. Vučković, G. S. Solomon, and Y. Yamamoto, Indistinguishable Photons from a SinglePhoton Device, Nature (London) 419, 594 (2002).

[11] A. Muller, E. B. Flagg, P. Bianucci, X. Y. Wang, D. G. Deppe, W. Ma, J. Zhang, G. J. Salamo, M. Xiao, and C. K. Shih, Resonance Fluorescence from a Coherently Driven Semiconductor Quantum Dot in a Cavity, Phys. Rev. Lett. 99, 187402 (2007).

[12] Paul A. Dalgarno, Jason M. Smith, Jamie McFarlane, Brian D. Gerardot, Kahled Karrai, Antonio Badolato, Pierre M. Petroff, and Richard J. Warburton, Coulomb Interactions in Single Charged Self-Assembled Quantum Dots: Radiative Lifetime and Recombination Energy, Phys. Rev. B 77, 245311 (2008).

[13] Kerry J. Vahala, Optical Microcavities, Nature (London) 424, 839 (2003).

[14] Julien Claudon, Joël Bleuse, Nitin Singh Malik, Maela Bazin, Périne Jaffrennou, Niels Gregersen, Christophe Sauvan, Philippe Lalanne, and Jean-Michel Gérard, A Highly Efficient Single-Photon Source Based on a Quantum Dot in a Photonic Nanowire, Nat. Photonics 4, 174 (2010).

[15] Alexander Högele, Stefan Seidl, Martin Kroner, Khaled Karrai, Richard J. Warburton, Brian D. Gerardot, and Pierre M. Petroff, Voltage-Controlled Optics of a Quantum Dot, Phys. Rev. Lett. 93, 217401 (2004).

[16] Mete Atatüre, Jan Dreiser, Antonio Badolato, Alexander Högele, Khaled Karrai, and Atac Imamoglu, QuantumDot Spin-State Preparation with Near-Unity Fidelity, Science 312, 551 (2006).

[17] J. Houel, A. V. Kuhlmann, L. Greuter, F. Xue, M. Poggio, B. D. Gerardot, P. A. Dalgarno, A. Badolato, P. M. Petroff, A. Ludwig, D. Reuter, A. D. Wieck, and R. J. Warburton, Probing Single-Charge Fluctuations at a GaAs/AlAs Interface Using Laser Spectroscopy on a Nearby InGaAs Quantum Dot, Phys. Rev. Lett. 108, 107401 (2012).

[18] Andreas V. Kuhlmann, Julien Houel, Arne Ludwig, Lukas Greuter, Dirk Reuter, Andreas D. Wieck, Martino Poggio, and Richard J. Warburton, Charge Noise and Spin Noise in a Semiconductor Quantum Device, Nat. Phys. 9, 570 (2013).

[19] Richard J. Warburton, Single Spins in Self-Assembled Quantum Dots, Nat. Mater. 12, 483 (2013).

[20] S. T. Yilmaz, P. Fallahi, and A. Imamoğlu, Quantum-DotSpin Single-Photon Interface, Phys. Rev. Lett. 105, 033601 (2010).

[21] K. De Greve, L. Yu, P. L. McMahon, J.S. Pelc, C. M. Natarajan, N. Y. Kim, E. Abe, S. Maier, C. Schneider, M. Kamp, S. Höfling, R. H. Hadfield, A. Forchel, M. M. Fejer, and Y. Yamamoto, Quantum-Dot Spin-Photon Entanglement via Frequency Downconversion to Telecom Wavelength, Nature (London) 491, 421 (2012).

[22] W. B. Gao, P. Fallahi, E. Togan, J. Miguel-Sanchez, and A. Imamoglu, Observation of Entanglement between a Quantum Dot Spin and a Single Photon, Nature (London) 491, 426 (2012).

[23] R. J. Warburton, C. Schulhauser, D. Haft, C. Schäflein, K. Karrai, J. M. Garcia, W. Schoenfeld, and P. M. Petroff, Giant Permanent Dipole Moments of Excitons in Semiconductor Nanostructures, Phys. Rev. B 65, 113303 (2002). 
[24] A. J. Bennett, M. A. Pooley, R. M. Stevenson, M. B. Ward, R. B. Patel, A. Boyer de la Giroday, N. Sköld, I. Farrer, C. A. Nicoll, D. A. Ritchie, and A. J. Shields, Electric-Field-Induced Coherent Coupling of the Exciton States in a Single Quantum Dot, Nat. Phys. 6, 947 (2010).

[25] S. Seidl, M. Kroner, A. Högele, K. Karrai, R. J. Warburton, A. Badolato, and P. M. Petroff, Effect of Uniaxial Stress on Excitons in a Self-Assembled Quantum Dot, Appl. Phys. Lett. 88, 203113 (2006).

[26] K. D. Jöns, R. Hafenbrak, R. Singh, F. Ding, J. D. Plumhof, A. Rastelli, O. G. Schmidt, G. Bester, and P. Michler, Dependence of the Redshifted and Blueshifted Photoluminescence Spectra of Single $\operatorname{In}_{x} \mathrm{Ga}_{1-x} \mathrm{As} / \mathrm{GaAs}$ Quantum Dots on the Applied Uniaxial Stress, Phys. Rev. Lett. 107, 217402 (2011).

[27] Benito Alén, Florian Bickel, Khaled Karrai, Richard J. Warburton, and Pierre M. Petroff, Stark-Shift Modulation Absorption Spectroscopy of Single Quantum Dots, Appl. Phys. Lett. 83, 2235 (2003).

[28] K. Karrai and R. J. Warburton, Optical Transmission and Reflection Spectroscopy of Single Quantum Dots, Superlattices Microstruct. 33, 311 (2003).

[29] N. Akopian, R. Trotta, E. Zallo, S. Kumar, P. Atkinson, A. Rastelli, O. G. Schmidt, and V. Zwiller, An Artificial Atom Locked to Natural Atoms, arXiv:1302.2005.

[30] V. M. Acosta, C. Santori, A. Faraon, Z. Huang, K.-M. C. $\mathrm{Fu}$, A. Stacey, D. A. Simpson, K. Ganesan, S. Tomljenovic-Hanic, A.D. Greentree, S. Prawer, and R.G. Beausoleil, Dynamic Stabilization of the Optical Resonances of Single Nitrogen-Vacancy Centers in Diamond, Phys. Rev. Lett. 108, 206401 (2012).

[31] Clemens Matthiesen, Anthony Nickolas Vamivakas, and Mete Atatüre, Subnatural Linewidth Single Photons from a Quantum Dot, Phys. Rev. Lett. 108, 093602 (2012).

[32] Andreas V. Kuhlmann, Julien Houel, Daniel Brunner, Arne Ludwig, Dirk Reuter, Andreas D. Wieck, and Richard J. Warburton, A Dark-Field Microscope for Background-Free Detection of Resonance Fluorescence from Single Semiconductor Quantum Dots Operating in a Set-and-Forget Mode, Rev. Sci. Instrum. 84, 073905 (2013).

[33] R. J. Warburton, C. Schäflein, D. Haft, F. Bickel, A. Lorke, K. Karrai, J. M. Garcia, W. Schoenfeld, and P. M. Petroff, Optical Emission from a Charge-Tunable Quantum Ring, Nature (London) 405, 926 (2000).

[34] C. Latta, A. Högele, Y. Zhao, A. N. Vamivakas, P. Maletinsky, M. Kroner, J. Dreiser, I. Carusotto, A. Badolato, D. Schuh, W. Wegscheider, M. Atature, and A. Imamoglu, Confluence of Resonant Laser Excitation and Bidirectional Quantum-Dot Nuclear-Spin Polarization, Nat. Phys. 5, 758 (2009).

[35] Warren G. Nagourney, Quantum Electronics for Atomic Physics (Oxford University Press, New York, 2010).

[36] Sh. Kogan, Electronic Noise and Fluctuations in Solids (Cambridge University Press, Cambridge, England, 1996).

[37] Rodney Loudon, The Quantum Theory of Light (Oxford University Press, New York, 2010), p. 352-360.

[38] B. Alén, A. Högele, M. Kroner, S. Seidl, K. Karrai, R. J. Warburton, A. Badolato, G. Medeiros-Ribeiro, and P. M. Petroff, Absorptive and Dispersive Optical Responses of Excitons in a Single Quantum Dot, Appl. Phys. Lett. 89, 123124 (2006).

[39] M. Atatüre, J. Dreiser, A. Badolato, and A. Imamoglu, Observation of Faraday Rotation from a Single Confined Spin, Nat. Phys. 3, 101 (2007). 